\title{
Adiposity and the isotemporal substitution of physical activity, sedentary time and sleep among school-aged children: a compositional data analysis approach
}

\author{
Dorothea Dumuid ${ }^{1 *}$ D, Tyman E. Stanford ${ }^{2,10}$, Željko Pedišić ${ }^{3}$, Carol Maher ${ }^{1}$, Lucy K. Lewis ${ }^{1,4}$, \\ Josep-Antoni Martín-Fernández ${ }^{5}$, Peter T. Katzmarzyk ${ }^{6}$, Jean-Philippe Chaput ${ }^{7}$, Mikael Fogelholm ${ }^{8}$, \\ Martyn Standage ${ }^{9}$, Mark S. Tremblay ${ }^{7}$ and Timothy Olds ${ }^{1}$
}

\begin{abstract}
Background: Daily activity data are by nature compositional data. Accordingly, they occupy a specific geometry with unique properties that is different to standard Euclidean geometry. This study aimed to estimate the difference in adiposity associated with isotemporal reallocation between daily activity behaviours, and to compare the findings from compositional isotemporal subsitution to those obtained from traditional isotemporal substitution.

Methods: We estimated the differences in adiposity (body fat\%) associated with reallocating fixed durations of time (isotemporal substitution) between accelerometer-measured daily activity behaviours (sleep, sedentary time and light and moderate-to-vigorous physical activity (MVPA)) among 1728 children aged 9-11 years from Australia, Canada, Finland and the UK (International Study of Childhood Obesity, Lifestyle and the Environment, 2011-2013). We generated estimates from compositional isotemporal substitution models and traditional non-compositional isotemporal substitution models.
\end{abstract}

Results: Both compositional and traditional models estimated a positive (unfavourable) difference in body fat\% when time was reallocated from MVPA to any other behaviour. Unlike traditional models, compositional models found the differences in estimated adiposity (1) were not necessarily symmetrical when an activity was being displaced, or displacing another (2) were not linearly related to the durations of time reallocated, and (3) varied depending on the starting composition.

Conclusion: The compositional isotemporal model caters for the constrained and therefore relative nature of activity behaviour data and enables all daily behaviours to be included in a single statistical model. The traditional model treats data as real variables, thus the constrained nature of time is not accounted for, nor reflected in the findings. Findings from compositional isotemporal substitution support the importance of MVPA to children's health, and suggest that while interventions to increase MVPA may be of benefit, attention should be directed towards strategies to avoid decline in MVPA levels, particularly among already inactive children. Future applications of the compositional model can extend from pair-wise reallocations to other configurations of time-reallocation, for example, increasing MVPA at the expense of multiple other behaviours.

Keywords: Physical activity, sedentary behaviour, sleep, isotemporal substitution, adiposity

\footnotetext{
* Correspondence: dorothea.dumuid@mymail.unisa.edu.au

${ }^{1}$ Alliance for Research in Exercise, Nutrition and Activity (ARENA), School of Health Sciences, University of South Australia, GPO Box 2471, Adelaide, SA 5001, Australia

Full list of author information is available at the end of the article
} 


\section{Background}

Higher levels of moderate-to-vigorous physical activity (MVPA) and sleep duration in children have been associated with lower adiposity (e.g., body fat\%); conversely, greater sedentary time has been associated with higher adiposity [1-3]. More recently, studies have examined the associations between adiposity and the reallocation of a fixed duration of one activity behaviour for another, using the isotemporal substitution methodology proposed by Mekary et al. [4]. Conceptually, the traditional isotemporal substitution paradigm takes the finite nature of daily activity behaviours into account. However, the suitability of this isotemporal model for constrained data has been questioned $[5,6]$.

The traditional isotemporal substitution method is based on regression modeling. All activity behaviours ( $\mathrm{min} /$ day) except one are used as explanatory variables in the model, and a term for the total number of measured minutes, i.e. the sum of all activity behaviour variables, is also included. The non-standardised regression coefficient corresponding to each of the activity behaviours included as explanatory variables in the model is considered to be the estimated change in the outcome variable when one minute of that activity behaviour replaces the activity behaviour excluded from the regression model. A more detailed explanation of the traditional isotemporal substitution model can be found elsewhere $[4,7]$.

An alternative approach for analysing daily activity behaviours - compositional data analysis - was recently suggested by Pedišić [6] and later pioneered in empirical studies by Chastin et al. [8] and Carson et al. [9]. Compositional data analysis conceptualises individuals' daily activity data as compositions, made up of mutually exclusive and exhaustive parts (times spent in sleep, sedentary behaviour, light physical activity (LPA) and MVPA), which are constrained by a daily constant sum of $1440 \mathrm{~min}$. Accordingly, these data exist in a constrained space (the simplex) governed by a specific geometry (Aitchison geometry) [10]. Operations in the simplex differ to those defined for real space, i.e., Euclidean geometry (e.g., the Euclidean sum and product are operationalised as perturbation and powering in the simplex) [10]. Therefore traditional statistical techniques using Euclidean operations (such as multiple linear regression) are incongruent with the Aitchison geometry of the simplex [11]. Compositions can, however be translated to real space through the application of isometric log ratio (ilr) coordinate systems [11]. Once compositions are represented as ilr coordinates, they are governed by Euclidean geometry, and traditional analytical techniques such as linear regression can be used [11].

The ilr linear multiple regression model can be used to estimate or predict an outcome of any given composition that has been expressed as ilr coordinates [12]. By systematically changing the predictive composition (as ilr coordinates) to simulate isotemporal reallocation between activity behaviourpairs, a series of estimated outcomes can be generated. Estimated adiposity can be calculated for (1) a baseline composition (e.g., the mean composition), and (2) for a new composition, where a set duration of time has been reallocated from one activity behaviour to another, keeping the remaining behaviours constant at their baseline values. The difference in estimated adiposity can then be calculated for the two compositions. The compositional isotemporal substitution technique is not limited to creating estimates only for the substitution of one behaviour (e.g., sedentary time) for one other behaviour (e.g., MVPA), but the behaviour of interest could be substituted by two or more other behaviours (e.g., MVPA and sleep). In fact, the difference in adiposity estimated for any reallocation between daily activity behaviours (eg., sleep, sedentary time, LPA and MVPA) can be assessed. This approach was developed by Dumuid and colleagues [13] and first applied in Fairclough et al [14] on a sample of 169 grade five children in the United Kingdom. It is unknown if and how the results from compositional and traditional isotemporal substitution approaches differ.

This study is the first to estimate the difference in adiposity associated with reallocations of time between daily activity behaviours among children using compositional and traditional isotemporal substitution methods, and to compare the findings obtained from the two approaches.

\section{Methods \\ Participants}

Participants were from four study sites (Australia, Canada, United Kingdom and Finland) included in the larger cross-sectional 12-nation International Study of Childhood Obesity, Lifestyle and the Environment (ISCOLE) [15]. The four sites were chosen based on previous findings suggesting relative homogeneity of lifestyle behaviours among children at these sites [16]. Site investigators aimed to recruit a sex-balanced sample of 500 children from each site, based on a priori power calculations [15]. Children aged 9-11 years $(n=2156)$ were recruited from schools in urban and suburban settings, and stratified by socioeconomic status [15]. Data were collected between September 2011 and December 2013. Children were excluded if they had incomplete data for any of the following parameters; activity behaviours $(n=319)$, as well as body fat $\%(n=28)$ and covariates (highest education level of either parent, number of parents and siblings in the home; $n=81$ ), resulting in a final sample of 1728 children.

\section{Ethics}

The Pennington Biomedical Research Center in Baton Rouge, Louisiana coordinated ISCOLE and received ethical approval for the overall study protocol from their Institutional Review Board. The four sites also received approval from local ethics committees and school boards. Parents 
provided signed informed consent and child assent was attained prior to inclusion in the study. ISCOLE is registered on ClinicalTrials.gov, Identifier: NCT01722500.

\section{Daily activity behaviours}

Activity behaviours were measured during the school year over 7 days, by 24-h accelerometry. Actigraph GT3X+ Accelerometers (ActiGraph LLC, Pensacola, FL) were worn on the right hip, with instruction to remove the device only for water-based activities. Wear time compliance in ISCOLE was high (daily average $=22.8 \mathrm{~h}$ ). Participants were required to have at least 4 days of valid accelerometry data (with $\geq 10 \mathrm{~h}$ of waking wear/day, including at least one weekend day [17]). Data were downloaded in 1-s epochs using the low-frequency extension filter (ActiLife software version 5.6). A fully automated algorithm was used to estimate nocturnal sleep duration (from data aggregated to 60s epochs) $[18,19]$. Waking behaviours were determined (from data aggregated to 15-s epochs), once nocturnal sleep time and awake non-wear time (any sequence of $\geq 20$ consecutive minutes of 0 activity counts) were removed. Evenson's cut-points were used to define sedentary time $(\leq 25$ counts per $15 \mathrm{~s}$ ), LPA (26-573 counts per $15 \mathrm{~s}$ ), and MVPA ( $\geq 574$ counts per $15 \mathrm{~s}$ ) [20]. These cut-points have been shown to provide acceptable classification accuracy for 515 year old children [21]. The daily average for each part of the composition (sleep, sedentary time, LPA and MVPA) was weighted for week:weekend days at 5:2.

\section{Adiposity}

Body fat $\%$ was used to indicate children's overall adiposity. Body fat $\%$ was measured during school visits using a portable Tanita SC-240 bioelectrical impedance scale (TANITA Corporation, Tokyo, Japan) [22]. Two measurements were taken, and the average was used in analysis (if measurements were $>2.0 \%$ apart, a third measurement was taken, and the closest two were averaged for analysis). Bioelectrical impedance analysis is a valid $(\mathrm{r}=0.69-0.79)$ and reliable $\left(\mathrm{CV}_{\text {intra }}=\right.$ $3 \%, \mathrm{SEM}_{\text {intra }}=-0.91$ to 1.61 body fat\%) estimate of body fat for school-age children compared to gold standards of underwater weighing and dual-energy X-ray absorptiometry (DXA) [23, 24]. The Tanita SC-240 has acceptable accuracy for estimating body fat $\%$ in this age group when assessed against DXA [22]. Body mass index (BMI) z-score, used to classify children into weight-status sub-groups, was calculated from measured height (Seca 213 portable stadiometer, Hamburg, Germany) and weight (TANITA Corporation, Tokyo, Japan [22]), using BMI = weight $(\mathrm{kg}) /$ height $\left(\mathrm{m}^{2}\right)$ and age- and sex-specific World Health Organization reference data [25] for standardisation as z-scores.

\section{Covariates}

The highest education level of either parent, and the number of parents and siblings in the home were reported by parents [15]. These sociodemographic factors were selected as covariates due to their potential influence on both activity behaviours and adiposity [26-28].

\section{Analysis}

Analysis was conducted in R ( $\mathrm{R}$ Development Core Team, Vienna, Austria), using the packages Compositions [29] and robCompositions [30]. The daily behaviour composition was described in terms of centre (geometric mean of each behaviour, adjusted to collectively sum to $1440 \mathrm{~min}$ ) $[8,31]$ and dispersion (variation matrix) $[8,10]$. Analysis consisted of exploring the relationship between isotemporal substitution of one behaviour for another behaviour and children's body fat\%, using (i) traditional isotemporal substitution [4] and (ii) compositional isotemporal substitution. All analyses were stratified by sex.

Traditional isotemporal substitution analysis followed the procedure outlined by Mekary et al. [4]. Four linear regression models were created with body fat $\%$ as the dependent variable. The explanatory variables of the first model included each daily behaviour (sedentary time, LPA, MVPA) except one (sleep). Total time was also included; with the effect of constraining the linear model so that the regression coefficients represented the estimated change in adiposity associated with the substitution of one unit of sleep (i.e., $30 \mathrm{~min}$ ) for one unit of the corresponding remaining behaviours. To capture all isotemporal eventualities, the remaining three linear models each omitted a different behaviour, while adjusting for all remaining behaviours, total wear time and covariates.

Compositional isotemporal substitution used multiple linear regression models with the activity behaviour composition (expressed as ilr coordinates) and sociodemographic covariates as explanatory variables. A comprehensive explanation of the compositional analysis and sample $\mathrm{R}$ code is included in Additional file 1. The ilr model was used to estimate body fat\% at a baseline composition (e.g., the mean daily activity behaviour composition), and subsequently at new compositions (e.g., sleep $=$ mean sleep +30 min, sedentary time $=$ mean sedentary $-30 \mathrm{~min}, \mathrm{LPA}=$ mean LPA and MVPA = mean MVPA). The estimated absolute differences in body fat percentage units was calculated using predictions from the model (i.e., estimated difference in body fat $\%=$ estimated body fat $\%$ at baseline composition - estimated body fat $\%$ at new composition). Estimates were calculated for time reallocations of $30 \mathrm{~min}$. Effect sizes (ES) were calculated by dividing the estimated differences by the mean standard deviation of body fat $\%$. Additionally, estimated changes in body fat $\%$ across the continuum of reallocations in 5-min increments up to $70 \mathrm{~min}$ were graphically illustrated, using MVPA as an example.

This method of compositional isotemporal substitution uses a different approach from the change-matrix procedure outlined in Chastin et al. [8], where pairwise log ratios 
obtained by applying the inverse ilr transformation to the regression coefficients are introduced to facilitate the interpretation of the change in response variable. Each of these pairwise log-ratios is analogous to one of the three ilr coordinates of a four-part composition. However, the remaining ilr will necessarily contain information regarding the two components involved in the pair-wise log ratio. The method in Chastin et al. [8] is a useful tool, but prediction and inference are more comprehensive when made using the complete set of ilr coordinates.

Compositional isotemporal substitution was conducted in sex-stratified cohorts, at the daily behaviour mean composition (geometric mean of each behaviour, adjusted to collectively sum to the daily total of $1440 \mathrm{~min}$ ) for all children, and repeated at the daily mean composition for (i) normal weight children $(-2 \leq \mathrm{zBMI} \leq 1)$; (ii) overweight children $(1<\mathrm{zBMI} \leq 2)$; and (iii) obese children $(\mathrm{zBMI}>2)$ [25].

\section{Results}

Included participants were more likely to be girls $(P=0.02)$, have parents of higher education $(P=0.04)$ and fewer siblings $(P<0.001)$ than excluded participants (Additional file 2$)$.
They also had different mean activity behaviour compositions $(P<0.001)$, but not body fat\% $(P=0.27)$. Daily averages of sleep, sedentary time, LPA and MVPA for boys and girls and variation matrices describing relative dispersion among pairwise behaviours are included in Additional file 3 .

\section{Traditional isotemporal substitution}

The absolute differences in estimated body fat $\%$ appeared to be the greatest when time was reallocated between LPA and MVPA (boys) or either LPA or sedentary time and MVPA (girls) (Table 1).

\section{Compositional isotemporal substitution}

For both sexes, higher estimated body fat $\%$ was associated with the reallocation of time from MVPA to other behaviours (Table 1). Notably, the estimated difference in adiposity was not exactly symmetrical, e.g., a reallocation of $30 \mathrm{~min}$ from MVPA to sedentary time was associated with a larger estimated absolute difference in body fat\% $(+2.31 \mathrm{ES}=0.36$ for boys; +2.94 ES 0.41 for girls) than when $30 \mathrm{~min}$ was reallocated from sedentary to MVPA $(-1.54 \mathrm{ES}=0.24$ for

Table 1 Traditional and compositional isotemporal substitution: Estimated absolute differences in body fat\% associated with 30-min pair-wise reallocations between activity behaviours

\begin{tabular}{|c|c|c|c|c|c|c|c|c|c|}
\hline \multicolumn{10}{|l|}{ BOYS $n=760$} \\
\hline \multirow[t]{2}{*}{ Model } & Body fat $\%$ & Sleep & & SED & & LPA & & MVPA & \\
\hline & $30 \mathrm{~min}$ & $\Delta^{\prime}$ & $95 \% \mathrm{Cl}$ & $\Delta^{\prime}$ & $95 \% \mathrm{Cl}$ & $\Delta^{\prime}$ & $95 \% \mathrm{Cl}$ & $\Delta^{\prime}$ & $95 \% \mathrm{Cl}$ \\
\hline Traditional & Sleep & & & -0.29 & $-0.56,-0.01$ & -0.56 & $-0.92,-0.20$ & 1.38 & $0.78,2.00$ \\
\hline Compositional & & & & -0.20 & $-0.46,0.05$ & -0.53 & $-0.87,-0.18$ & 2.11 & $1.37,2.85$ \\
\hline Traditional & SED & 0.29 & $0.01,0.56$ & & & -0.27 & $-0.58,0.03$ & 1.67 & $1.13,2.22$ \\
\hline Compositional & & 0.20 & $-0.05,0.46$ & & & -0.33 & $-0.63,-0.02$ & 2.31 & $1.62,2.99$ \\
\hline Traditional & LPA & 0.56 & $0.20,0.92$ & 0.27 & $-0.03,0.58$ & & & 1.95 & $1.26,2.63$ \\
\hline Compositional & & 0.49 & $0.16,0.83$ & 0.28 & $-0.01,0.57$ & & & 2.60 & $1.78,3.42$ \\
\hline Traditional & MVPA & -1.38 & $-2.00,-0.78$ & -1.67 & $-2.22,-1.13$ & -1.95 & $-2.63,-1.26$ & & \\
\hline Compositional & & -1.33 & $-1.83,-0.82$ & -1.54 & $-1.99,-1.09$ & -1.86 & $-2.46,-1.26$ & & \\
\hline \multicolumn{10}{|l|}{ GIRLS $n=968$} \\
\hline \multirow[t]{2}{*}{ Model } & Body fat $\%$ & Sleep & & SED & & LPA & & MVPA & \\
\hline & $30 \mathrm{~min}$ & $\Delta^{\prime}$ & $95 \% \mathrm{Cl}$ & $\Delta^{\prime}$ & $95 \% \mathrm{Cl}$ & $\Delta^{\prime}$ & $95 \% \mathrm{Cl}$ & $\Delta^{\prime}$ & $95 \% \mathrm{Cl}$ \\
\hline Traditional & Sleep & & & -0.55 & $-0.85,-0.25$ & -0.49 & $-0.85,-0.12$ & 1.60 & $0.80,2.39$ \\
\hline Compositional & & & & -0.52 & $-0.80,-0.24$ & -0.49 & $-0.85,-0.12$ & 2.44 & $1.34,3.54$ \\
\hline Traditional & SED & 0.55 & $0.25,0.85$ & & & 0.07 & $-0.25,0.38$ & 2.15 & $1.41,2.89$ \\
\hline Compositional & & 0.52 & $0.24,0.80$ & & & 0.02 & $-0.30,0.34$ & 2.94 & $1.89,4.00$ \\
\hline Traditional & LPA & 0.49 & $0.12,0.85$ & -0.07 & $-0.38,0.25$ & & & 2.08 & $1.20,2.97$ \\
\hline Compositional & & 0.47 & $0.13,0.82$ & -0.06 & $-0.36,0.24$ & & & 2.90 & $1.70,4.10$ \\
\hline Traditional & MVPA & -1.60 & $-2.39,-0.80$ & -2.15 & $-2.89,-1.41$ & -2.08 & $-2.97,-1.20$ & & \\
\hline Compositional & & -1.24 & $-1.88,-0.61$ & -1.78 & $-2.36,-1.19$ & -1.74 & $-2.50,-0.99$ & & \\
\hline
\end{tabular}

Abbreviations: $\Delta^{\prime}$ estimated difference in body fat\%, Cl confidence interval, SED sedentary time, LPA light physical activity, MVPA moderate-to-vigorous physical activity. Analysis adjusted for parental education level and number of parents and siblings. Note: Difference in body fat\% is estimated for the reallocation of time from the behaviour in the column to the behaviour in the row, i.e., the first value of -0.29 in Row 1 is the estimated difference in body fat $\%$ for the reallocation of $30 \mathrm{~min}$ from sedentary time to sleep among boys. Boys' mean daily activity composition (min/day): Sleep $=550 ; \mathrm{SED}=510 ; \mathrm{LPA}=308 ; \mathrm{MVPA}=72$. Mean body fat $\%$ for boys: $\bar{x}=18.1$. Girls' mean daily activity composition ( $\mathrm{min} /$ day): Sleep $=564$; SED $=520 ; \mathrm{LPA}=301 ; \mathrm{MVPA}=55$. Mean body fat $\%$ for girls: $\bar{x}=22.3$ 
boys; $-1.78 \mathrm{ES}=0.25$ for girls). For boys, the reallocation of any given time spent in MVPA to LPA tended to predict the largest positive (unfavourable) difference in body fat\% (Fig. 1), whereas for girls, the reallocation of any given time spent in MVPA to either sedentary time or LPA appeared to predict the largest positive difference in body fat\% (Fig. 2).

Reallocations modeled at the mean daily behaviour composition for different weight status subgroups (Additional file 4) demonstrated larger differences in estimated body fat $\%$ for overweight and obese children, compared to those classified as 'normal weight', particularly when the reallocated behaviour was MVPA (Figs. 3 and 4).

\section{Comparison between traditional and compositional isotemporal substitution}

Both statistical techniques found that the largest differences in estimated body fat\% were associated with reallocations of time away from MVPA. However, there were some important differences between the findings from the two approaches. The relative difference between the estimates for 30 -min reallocations ranged from $0.2 \%$ to $121 \%$ (median $=18 \%$ ) (calculated as the absolute difference between estimates from traditional and compositional models divided by a pooled estimate from both models). Larger absolute differences between the traditional and compositional models seem to be associated with higher estimates. Compositional isotemporal substitution findings also differed from traditional isotemporal substitution findings in the following ways: (i) the amount of difference in estimated body fat $\%$ when a behaviour was higher or lower was not exactly symmetrical, (Figs. 5 and 6), (ii) the difference in estimated body fat\% was not linearly proportional to the amount of time reallocated (Figs. 5 and 6), and (iii) traditional isotemporal models indicated the estimated change in a continuous outcome was associated with same time reallocations irrespective of the starting/reference durations of activity behaviours (in other words, estimated change in body fat $\%$ was identical when $30 \mathrm{~min}$ of MVPA was reallocated from a starting duration of, for example, either $0 \mathrm{~min} /$ day or $150 \mathrm{~min} /$ day, whilst in contrast, estimates of change in outcome from compositional models were relative to a starting/reference composition, thus respecting the relative nature of compositional data [Figs. 3 and 4]).

\section{Discussion}

\section{Main findings}

This study estimated the difference in adiposity associated with the isotemporal substitution of daily activity behaviours. For both traditional and compositional models, the greatest differences in body fat $\%$ were estimated for the reallocation of time from MVPA to other behaviours, and vice versa. The estimated differences in body fat $\%$ for 30 min reallocations with MVPA were relatively modest, with standardised effect sizes ranging between 0.2 and 0.4 .

There appeared to be sex differences between the findings. Both models estimated favourable absolute differences in body composition when sedentary behaviour replaced LPA (significant only in the compositional model) among boys, but not among girls. Furthermore, among boys estimated body composition was not most favourable when MVPA replaced sedentary time, whereas it was among girls. This



Fig. 1 Boys: Estimated absolute difference in body fat $\%$ associated with pair-wise reallocations of time between MVPA and other behaviours. Abbreviations: SED, Sedentary Time; LPA, Light Physical Activity; MVPA, Moderate-to-Vigorous Physical Activity. Mean daily behaviour composition for boys ( $\mathrm{min} /$ day): Sleep $=550, \mathrm{SED}=510, \mathrm{LPA}=308, \mathrm{MVPA}=72$ 


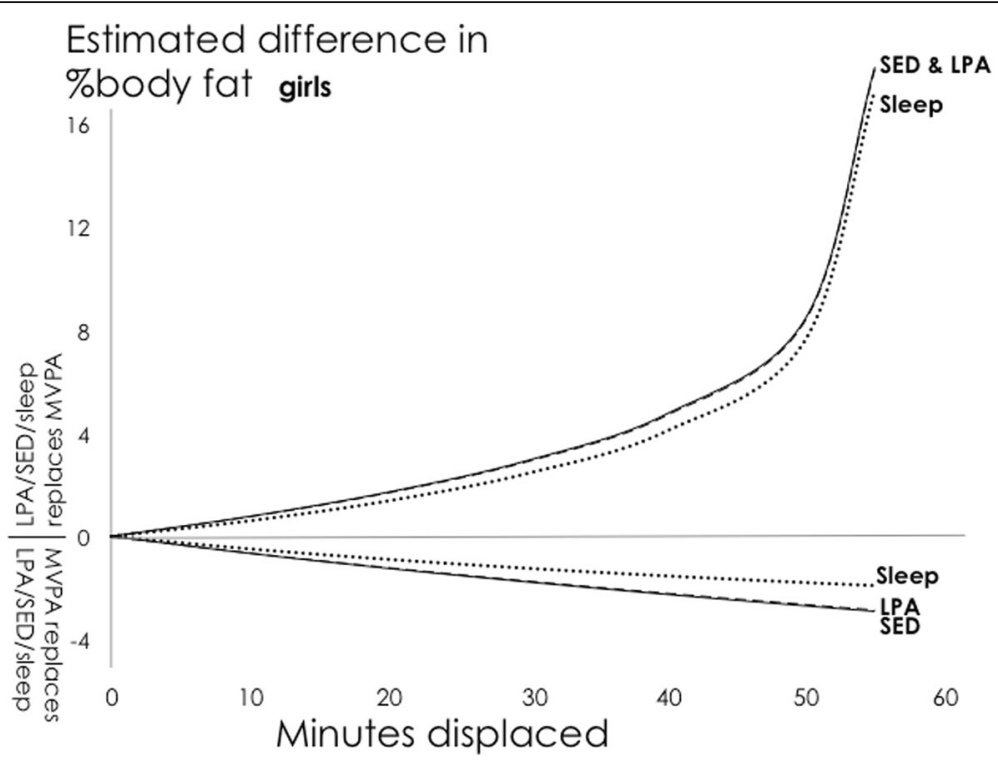

Fig. 2 Girls: Estimated absolute difference in body fat\% associated with pair-wise reallocations of time between MVPA and other behaviours. Abbreviations: SED, Sedentary Time; LPA, Light Physical Activity; MVPA, Moderate-to-Vigorous Physical Activity. Mean daily behaviour composition for girls (min/day): Sleep $=564, \operatorname{SED}=520, \mathrm{LPA}=301, \mathrm{MVPA}=55$

unexpected finding may be an artefact of the cross-sectional study design; there may be a sub-population of lean boys who accumulate large amounts of sedentary time. A number of previous studies have detected clusters of boys with high screen time [32-34]. Interestingly, membership of these clusters was not associated with higher adiposity.

There were some similarities between findings from traditional and compositional models, however there were fundamental differences between the two analytical approaches.
The distinguishing feature of the compositional approach is that the inherent compositional nature and corresponding geometry of the data are not violated by the mathematical operations applied [12].

\section{Differences between traditional and compositional methods}

Unlike traditional isotemporal substitution, the compositional method produces asymmetrical estimates. That is, a quantum

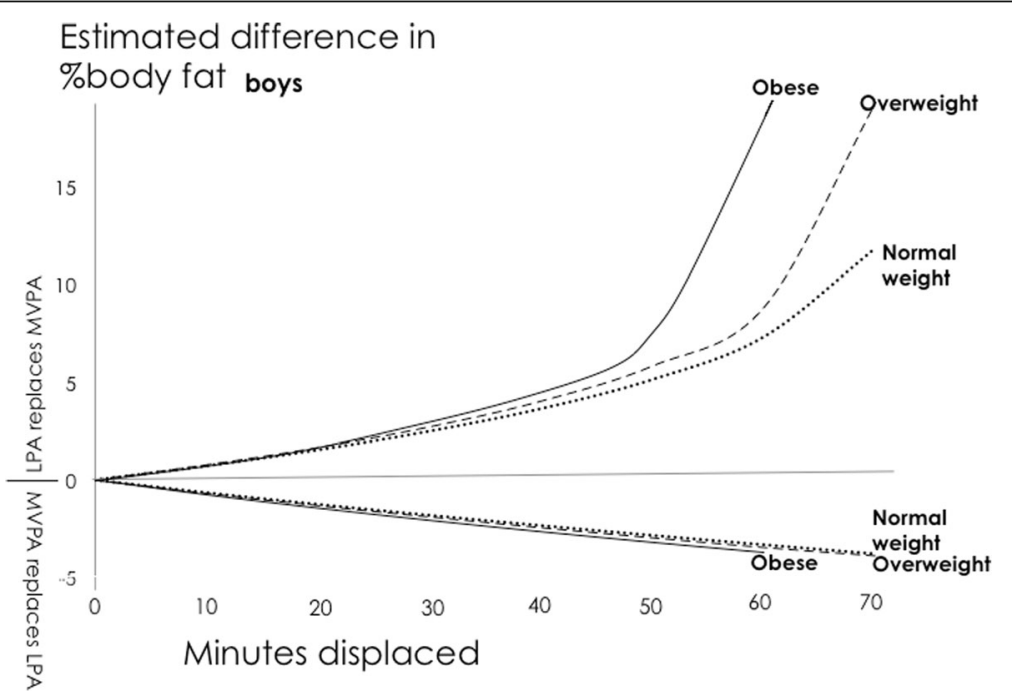

Fig. 3 Boys: Estimated absolute difference in body fat\% associated with pair-wise reallocations of time between MVPA and LPA around three different daily behaviour mean compositions; normal weight, overweight and obese. Abbreviations: LPA, Light Physical Activity; MVPA, Moderate-to-Vigorous Physical Activity. Mean boys' daily behaviour composition (min/day): Normal weight: Sleep $=551, S E D=506, L P A=307$, MVPA = 75; Overweight: Sleep =549, SED = 515, LPA = 307, MVPA = 70; Obese: Sleep =541, SED =526, LPA = 313, MVPA =61 




Fig. 4 Girls: Estimated absolute difference in body fat\% associated with pair-wise reallocations of time between MVPA and LPA around three different daily behaviour mean compositions; normal weight, overweight and obese. Abbreviations: LPA, Light Physical Activity; MVPA, Moderate-to-Vigorous Physical Activity. Mean girls' daily behaviour composition (min/day): Normal weight: Sleep =567, SED =515, LPA = 301, MVPA =57; Overweight: Sleep $=562$, SED $=526$, LPA $=298$, MVPA $=54$; Obese: Sleep $=543$, SED $=545$, LPA $=307$, MVPA $=45$

reallocation of one behaviour for another does not predict the exact inverse effect as the reverse reallocation. In the present study, asymmetry was most notably observed for reallocations with MVPA. Similar asymmetry with reallocation of MVPA was reported for other adiposity indicators by
Fairclough et al. [14]. Essentially, adding MVPA appears to yield lower returns, while reducing MVPA seems to be associated with higher expected differences in fatness. In addition, the amount of time reallocated between behaviours was not linearly related to the difference in estimated body fat $\%$.






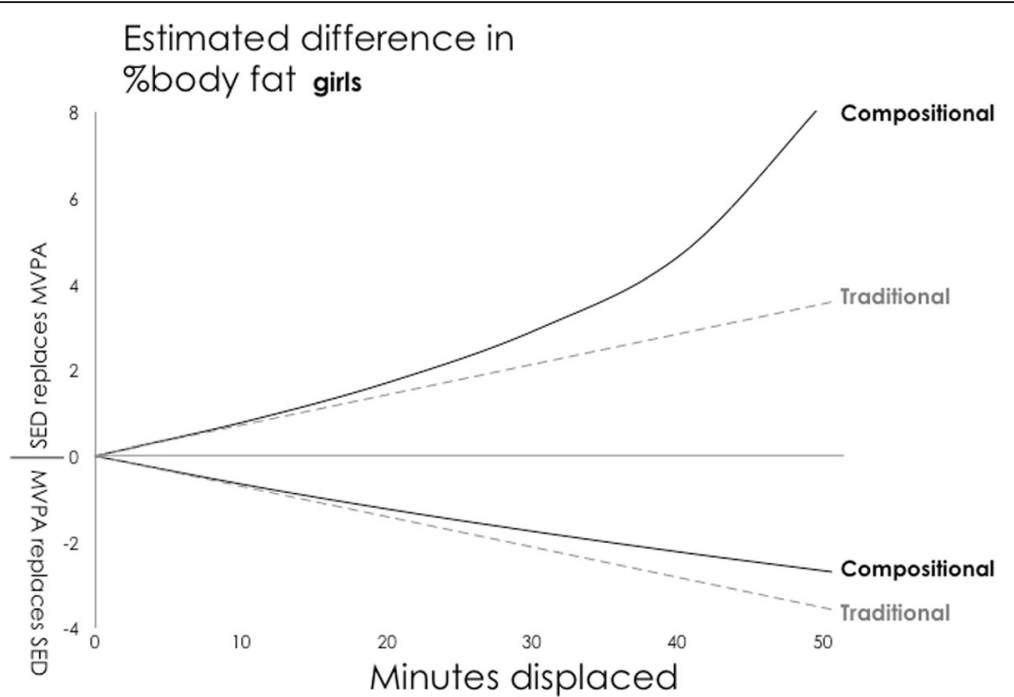

Fig. 6 Girls: Estimated absolute difference in body fat\% associated with pair-wise reallocations between MVPA and sedentary time as estimated by compositional and traditional isotemporal substitution. Abbreviations: SED, Sedentary Time; MVPA, LPA = Light Physical Activity; Moderate-to-Vigorous Physical Activity. Mean daily behaviour composition for girls (min/day): Sleep $=564$, SED $=520, L P A=301, M V P A=55$

These findings are consistent with dose-response curves observed in experimental studies (e.g. Sattelmair et al. [35]), where marginal increases in exposures regularly predict diminishing benefits [36]. The non-linearity of findings has two implications. Firstly, they suggest that adiposity interventions should encourage children (particularly those with low levels of physical activity) to be more physically active. Secondly, the asymmetry in the estimated dose-response curves suggests that interventions aimed at defending (i.e. maintaining) MVPA may be of great importance, even if MVPA is not increased. However, the cross-sectional nature of the findings and the relatively modest effect sizes should be noted when considering the implications of this study.

\section{Strengths and limitations}

This study has drawn on a large multi-national dataset using standardised measurements, including accelerometer-based measures of activity behaviours and objective measures of body fat $\%$ [15]. We used compositional data analysis techniques to quantify the adiposity association of isotemporal substitutions of activity behaviours.

Nonetheless, the study has several limitations. Accelerometers cannot distinguish completely between sitting and standing postures, with both potentially being classified as sedentary time. Whilst the analyses were adjusted for sociodemographic variables, there may be potential residual confounding from other lifestyle, social and environmental factors, such as diet, parental influence and perceived neighbourhood safety that were not included as covariates in our analyses. Furthermore, in ISCOLE, the participants were sampled from urban and suburban centres and were not nationally representative, limiting the generalisability of results [37]. Most importantly, due to the cross-sectional nature of the present isotemporal substitution analyses, findings are more correctly conceptualised as snapshots of adiposity associations with various daily behaviour compositions, than as adiposity associations of various behaviour substitutions within one day. It is therefore not possible to interpret the findings as the effect on adiposity for isotemporal substitutions of time between activity behaviours.

\section{Conclusions}

There were important differences between the two methods. Traditional isotemporal substitution did not account for (i) possible asymmetry of estimated changes in the outcome variables; (ii) possible non-linearity of the relationship between amount of time reallocated and estimated changes in the outcome variable, and (iii) possible differences in the relationships at various baseline daily activity levels. These differences stem from the fundamental limitation of traditional isotemporal substitution; it is a statistical technique for real space, requiring the use of standard operations and metrics to be applied in an Euclidean geometry. Daily activity behaviour data, being by nature compositional, do not exist in real space; rather they occupy a simplex sample space. The expression of daily activity behaviour data as ilr coordinates overcomes this statistical issue, as the corresponding coordinates occupy real space and follow a Euclidean geometry. Compositional data analysis provides a robust way of including all daily activity behaviours in multivariate statistical models, and thus can provide integrated insight into the associations with adiposity (and likely other health outcomes) of daily activity behaviours. Furthermore, the approach is not limited to pair- 
wise reallocations between behaviours; types of reallocation can be tailored to match the research question, for example, the reallocation of a fixed duration of time to MVPA at the expense of the remaining daily behaviours in a pro-rata fashion (one-for-many reallocation) can be used to explore the health associations of MVPA in relation to the remaining behaviours [12].

Compositional approaches are relatively new in analysing use-of-time data in health research. Future research should use compositional data analysis to explore reallocation relationships with other health outcomes, such as fitness, quality of life, cardiometabolic biomarkers and other disease risk indicators. It is particularly important to explore longitudinal changes in activity compositions associated with maturation, secular changes and interventions, and their relationships with indicators of health. The analyses conducted in the present study represent a basic approach to assessing the relationship between health and compositional isotemporal substitution. Once compositions have been expressed as ilr co-ordinates, more complex and potentially useful multivariate methods can be applied, for example, the use of splines or other non-parametric regression models. Furthermore, there is future scope to apply the compositional isotemporal substitution paradigm to other areas such as body composition and daily macronutrient intake.

\section{Additional files}

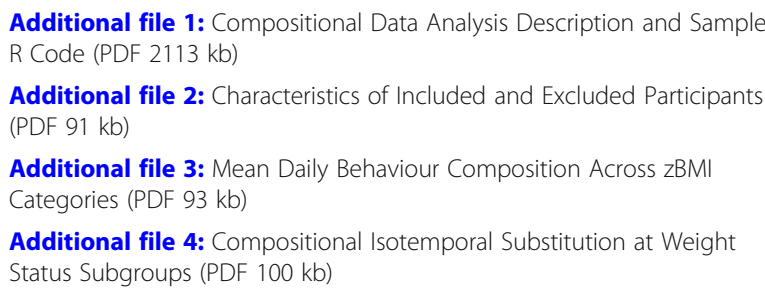

Additional file 3: Mean Daily Behaviour Composition Across zBMI Categories (PDF $93 \mathrm{~kb}$ )

Additional file 4: Compositional Isotemporal Substitution at Weight Status Subgroups (PDF $100 \mathrm{~kb}$ )

\section{Abbreviations}

BMl: Body Mass Index; DXA: Dual-energy X-ray absorptiometry;

ISCOLE: International Study of Childhood Obesity, Lifestyle and the Environment; LPA: Light Physical Activity; MVPA: Moderate-to-Vigorous Physical Activity

\section{Acknowledgements}

Not applicable

\section{Funding}

This work was supported by an Australian Government Research Training Program Scholarship [DD] and by The National Heart Foundation (100188) [CM]. This research has been partially supported by the Spanish Ministry of Economy and Competitiveness under the project CODA-RETOS (MTM2015-65016- C2-1(2)-R) [JAMF]. The ISCOLE study was funded by the Coca-Cola Company. The funders had no role in the design and conduct of the study, data collection, management, analysis and interpretation of the data; and decision to publish, preparation, review or approval of this manuscript. DD, TES, ZP and JAMF are not part of ISCOLE group.

\section{Availability of data and materials}

The data that support the findings of this study are available from Peter T. Katzmarzyk (Peter.Katzmarzyk@pbrc.edu) but restrictions apply to the availability of these data, which were used under license for the current study, and so are not publicly available. Data are however available from the authors upon reasonable request and with permission of Pennington Biomedical Research Center.

\section{Authors' contributions}

DD and TO conceived the idea for the study. DD, TO and CM constructed the study plan. CM, LKL, JPC, MF, MS, MST, PTK and TO planned and conducted the data collection. DD, TES, JAMF, TO and ZP contributed to the development of the data analysis design. DD and TES analysed the data. DD, ZP and TO drafted the text of the manuscript. CM, LKL, JPC, MF, MS, MST and PTK contributed to the writing of the manuscript. DD, TO, ZP, TES and JAMF drafted the rebuttal and revised the final draft of the manuscript. All authors read and approved the revised version of the manuscript.

\section{Ethics approval and consent to participate}

The Pennington Biomedical Research Center in Baton Rouge, Louisiana coordinated ISCOLE and received ethical approval for the overall study protocol from their Institutional Review Board. The four sites also received approval from local ethics committees and school boards. Parents provided signed informed consent and child assent was attained prior to inclusion in the study.

\section{Consent for publication}

Not applicable

\section{Competing interests}

The authors declare that they have no competing interests

\section{Publisher's Note}

Springer Nature remains neutral with regard to jurisdictional claims in published maps and institutional affiliations.

\section{Author details}

${ }^{1}$ Alliance for Research in Exercise, Nutrition and Activity (ARENA), School of Health Sciences, University of South Australia, GPO Box 2471, Adelaide, SA 5001, Australia. ${ }^{2}$ School of Mathematical Sciences, The University of Adelaide, Adelaide, Australia. ${ }^{3}$ Institute of Sport, Exercise and Active Living, Victoria University, Melbourne, Australia. ${ }^{4}$ School of Health Sciences, Flinders University and School of Health Sciences, University of South Australia, Adelaide, Australia. ${ }^{5}$ Department of Computer Science, Applied Mathematics and Statistics, University of Girona, Girona, Spain. ${ }^{6}$ Pennington Biomedical Research Center, Baton Rouge, USA. ${ }^{7}$ Healthy Active Living and Obesity Research, Children's Hospital of Eastern Ontario Research Institute, Ottawa, Canada. ${ }^{8}$ Department of Food and Environmental Sciences, University of Helsinki, Helsinki, Finland. ${ }^{9}$ Department for Health, University of Bath, Bath, England. ${ }^{10}$ LBT Innovations, Adelaide, Australia.

Received: 10 August 2017 Accepted: 22 February 2018 Published online: 02 March 2018

References

1. Poitras VJ, Gray CE, Borghese MM, Carson V, Chaput J-P, Janssen I, et al. Systematic review of the relationships between objectively measured physical activity and health indicators in school-aged children and youth 1. Appl Physiol Nutr Metab. 2016;41(6):S197-239.

2. Chaput J-P, Gray CE, Poitras VJ, Carson V, Gruber R, Olds T, et al. Systematic review of the relationships between sleep duration and health indicators in school-aged children and youth 1. Appl Physiol Nutr Metab. 2016;41(6):S266-S82.

3. Carson V, Hunter S, Kuzik N, Gray CE, Poitras VJ, Chaput J-P, et al. Systematic review of sedentary behaviour and health indicators in school-aged children and youth: an update 1. Appl Physiol Nutr Metab. 2016:41(6):S240-S65.

4. Mekary RA, Willett WC, Hu FB, Ding EL. Isotemporal substitution paradigm for physical activity epidemiology and weight change. Am J Epidemiol. 2009;170(4):519-27.

5. Gupta N, Heiden M, Aadahl M, Korshøj M, Jørgensen MB, Holtermann A. What Is the Effect on Obesity Indicators from Replacing Prolonged Sedentary Time with Brief Sedentary Bouts, Standing and Different Types of Physical Activity during Working Days? A Cross-Sectional AccelerometerBased Study among Blue-Collar Workers. PLoS One. 2016;11(5):e0154935.

6. Pedišić Ž. Measurement issues and poor adjustments for physical activity and sleep undermine sedentary behaviour research - the focus should shift to the balance between sleep, sedentary behaviour, standing and activity. Kinesiology. 2014;46(1):135-46. 
7. Pedišić Ž, Dumuid D, Olds T. Integrating sleep, sedentary behaviour, and physical activity research in the emerging field of time-use epidemiology: definitions, concepts, statistical methods, theoretical framework, and future directions. Kinesiology. 2017:49(2):252-69.

8. Chastin SF, Palarea-Albaladejo J, Dontje ML, Skelton DA. Combined effects of time spent in physical activity, sedentary behaviors and sleep on obesity and cardio-metabolic health markers: A novel compositional data analysis approach. PLoS One. 2015;10(10):e0139984.

9. Carson V, Tremblay MS, Chaput J-P, Chastin SF. Associations between sleep duration, sedentary time, physical activity, and health indicators among Canadian children and youth using compositional analyses 1. Appl Physiol Nutr Metab. 2016;41(6):S294-302.

10. Aitchison J. The statistical analysis of compositional data. J Royal Statistical Soc Series B (Methodological). 1982:139-77.

11. Mateu-Figueras G, Pawlowsky-Glahn V, Egozcue J. The Principle of Working on Coordinates. In: Pawlowsky-Glahn V, Buccianti A, editors. Compositional Data Analysis: Theory and Applications. Chichester: John Wiley \& Sons; 2011. p. 29-42.

12. Dumuid D, Stanford T, Martín-Fernández J, Pedišić Ž, Maher C, Lewis L, et al. Compositional data analysis for physical activity, sedentary time and sleep research. Stat Methods Med Res. 2017; https://doi.org/10.1177/ 0962280217710835.

13. Dumuid D, Pedišić Ž, Stanford TE, Martín-Fernández J-A, Hron K, Maher CA, et al. The compositional isotemporal substitution model: A method for estimating changes in a health outcome for reallocation of time between sleep, physical activity and sedentary behaviour. Stat Methods Med Res. 2017; https://doi.org/10.1177/0962280217737805.

14. Fairclough SJ, Dumuid D, Taylor S, Curry W, Bronagh M, Stratton G, et al. Fitness, fatness and the reallocation of time between children's daily movement behaviours: an analysis of compositional data. Int J Behav Nutr Phys Act. 2017;14(64) https://doi.org/10.1186/s12966-017-0521-z

15. Katzmarzyk PT, Barreira TV, Broyles ST, Champagne CM, Chaput J-P, Fogelholm M, et al. The international study of childhood obesity, lifestyle and the environment (ISCOLE): Design and methods. BMC Public Health. 2013;13(1):E900.

16. Dumuid D, Olds T, Lewis L, Martin-Fernández J, Barreira T, Broyles S, et al. The adiposity of children is associated with their lifestyle behaviours: a cluster analysis of school-aged children from 12 nations. Pediatr Obes. 2018; 13(2):111-9.

17. Tudor-Locke C, Barreira TV, Schuna JM, Mire EF, Chaput J-P, Fogelholm M, et al. Improving wear time compliance with a 24-hour waist-worn accelerometer protocol in the International Study of Childhood Obesity, Lifestyle and the Environment (ISCOLE). Int J Behav Nutr Phys Act. 2015;12(1):1-9.

18. Barreira TV, Schuna JM Jr, Mire EF, Katzmarzyk PT, Chaput J-P, Leduc G, et al. Identifying children's nocturnal sleep using 24-h waist accelerometry. Med Sci Sports Exerc. 2015;47(5):937-43.

19. Tudor-Locke C, Barreira TV, Schuna JM Jr, Mire EF, Katzmarzyk PT. Fully automated waist-worn accelerometer algorithm for detecting children's sleep-period time separate from 24-h physical activity or sedentary behaviors. Appl Physiol Nutr Metab. 2013;39(1):53-7.

20. Evenson KR, Catellier DJ, Gill K, Ondrak KS, McMurray RG. Calibration of two objective measures of physical activity for children. J Sports Sci. 2008;26(14):1557-65.

21. Trost SG, Loprinzi PD, Moore R, Pfeiffer KA Comparison of accelerometer cut points for predicting activity intensity in youth. Med Sci Sports Exerc. 2011:43(7):1360-8.

22. Barreira TV, Staiano AE, Katzmarzyk PT. Validity assessment of a portable bioimpedance scale to estimate body fat percentage in White and AfricanAmerican children and adolescents. Pediatr Obes. 2013;8(2):e29-32.

23. Jensky-Squires NE, Dieli-Conwright CM, Rossuello A, Erceg DN, McCauley S, Schroeder ET. Validity and reliability of body composition analysers in children and adults. Br J Nutr. 2008;100(04):859-65.

24. Elberg J, McDuffie J, Sebring N, Salaita C, Keil M, Robotham D, et al. Comparison of methods to assess change in children's body composition. Am J Clin Nutri. 2004:80:64-9.

25. de Onis M, Onyango A, Borghi E, Siyam A, Nishida C, Siekmann J. Development of a WHO growth reference for school-aged children and adolescents. Bull World Health Organ. 2007;85:660-7.

26. Shrewsbury V, Wardle J. Socioeconomic status and adiposity in childhood: a systematic review of cross-sectional studies 1990-2005. Obesity. 2008;16(2):275-84.

27. Gibson LY, Byrne SM, Davis EA, Blair E, Jacoby P, Zubrick SR. The role of family and maternal factors in childhood obesity. Med J Aust. 2007;186(11):591.

28. Crawford D, Cleland V, Timperio A, Salmon J, Andrianopoulos N, Roberts R, et al. The longitudinal influence of home and neighbourhood environments on children's body mass index and physical activity over 5 years: the CLAN study. Int J Obes. 2010;34(7):1177-87.

29. van den Boogaart KG, Tolosana-Delgado R. "Compositions": a unified R package to analyze compositional data. Comput Geosci. 2008:34(4):320-38.

30. Templ M, Hron K, Filzmoser P. robCompositions: an R-package for robust statistical analysis of compositional data. In: Pawlowsky-Glahn V, Buccianti A, editors. Compositional Data Analysis: Theory and Applications. Chichester: John Wiley \& Sons, Ltd; 2011. p. 341-55.

31. Martín-Fernández J-A, Daunis-i-Estadella J, Mateu-Figueras G. On the interpretation of differences between groups for compositional data. SORTStatistics and Operations Research Transactions. 2015:39(2):231-52.

32. Veloso SM, Matos MG, Carvalho M, Diniz JA. Psychosocial factors of different health behaviour patterns in adolescents: association with overweight and weight control behaviours. J Obes. 2012;2012

33. Nuutinen $T$, Lehto $E$, Ray $C$, Roos $E$, Villberg J, Tynjälä J. Clustering of energy balance-related behaviours, sleep, and overweight among Finnish adolescents. Int J Pub Health. 2017:1-10.

34. Te Velde SJ, De Bourdeaudhuij I, Thorsdottir I, Rasmussen M, Hagströmer M, Klepp K-I, et al. Patterns in sedentary and exercise behaviors and associations with overweight in 9-14-year-old boys and girls-a crosssectional study. BMC Public Health. 2007;7(1):16.

35. Sattelmair J, Pertman J, Ding EL, Kohl HW, Haskell W, Lee I-M. Dose response between physical activity and risk of coronary heart disease: A meta analysis. Circulation. 2011:124(7):789-95.

36. Pate RR, Pratt M, Blair SN, Haskell WL, Macera CA, Bouchard C, et al. Physical activity and public health: a recommendation from the Centers for Disease Control and Prevention and the American College of Sports Medicine. JAMA. 1995;273(5):402-7.

37. LeBlanc AG, Katzmarzyk PT, Barreira TV, Broyles ST, Chaput J-P, Church TS, et al. Are participant characteristics from ISCOLE study sites comparable to the rest of their country? Int J Obes Suppl. 2015;5:S9-S16.

\section{Submit your next manuscript to BioMed Central and we will help you at every step:}

- We accept pre-submission inquiries

- Our selector tool helps you to find the most relevant journal

- We provide round the clock customer support

- Convenient online submission

- Thorough peer review

- Inclusion in PubMed and all major indexing services

- Maximum visibility for your research

Submit your manuscript at www.biomedcentral.com/submit
) Biomed Central 